PREPARED FOR THE U.S. DEPARTMENT OF ENERGY, UNDER CONTRACT DE-AC02-76CH03073

PPPL-3958

PPPL-3958

UC-70

Measurement of the Internal Magnetic Field of Plasmas using an Alpha Particle Source

by

S.J. Zweben, D.S. Darrow, P.W. Ross, J.L. Lowrance and G. Renda

May 2004

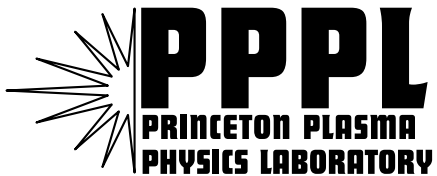

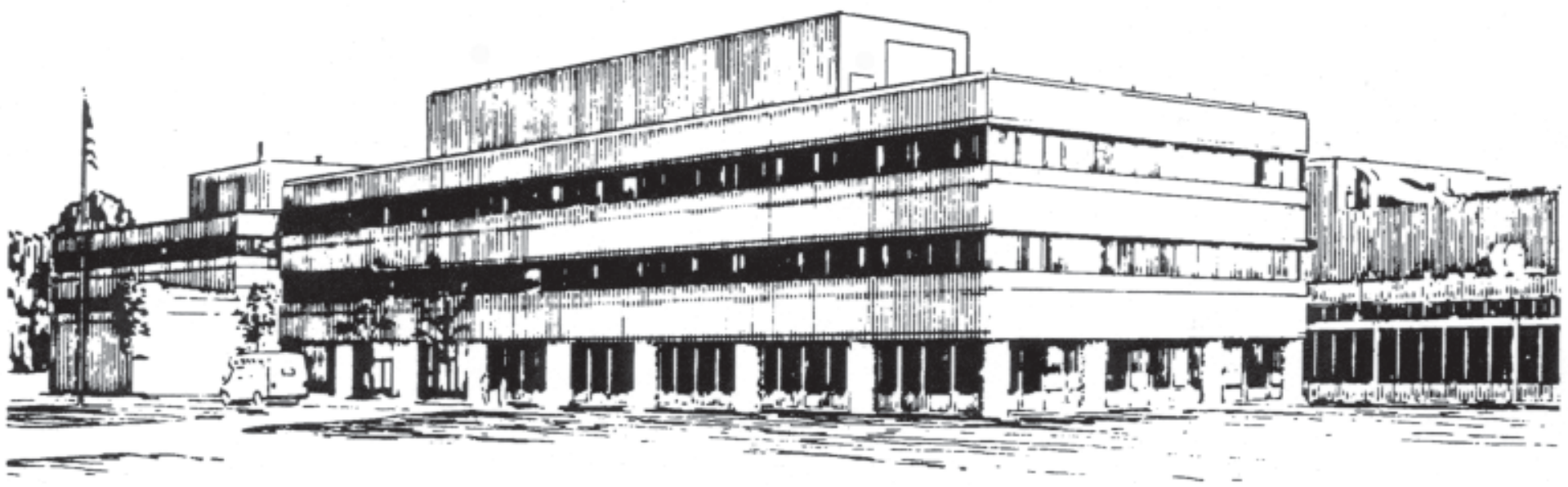

PRINCETON PLASMA PHYSICS LABORATORY PRINCETON UNIVERSITY, PRINCETON, NEW JERSEY 


\section{PPPL Reports Disclaimer}

This report was prepared as an account of work sponsored by an agency of the United States Government. Neither the United States Government nor any agency thereof, nor any of their employees, makes any warranty, express or implied, or assumes any legal liability or responsibility for the accuracy, completeness, or usefulness of any information, apparatus, product, or process disclosed, or represents that its use would not infringe privately owned rights. Reference herein to any specific commercial product, process, or service by trade name, trademark, manufacturer, or otherwise, does not necessarily constitute or imply its endorsement, recommendation, or favoring by the United States Government or any agency thereof. The views and opinions of authors expressed herein do not necessarily state or reflect those of the United States Government or any agency thereof.

\section{Availability}

This report is posted on the U.S. Department of Energy's Princeton Plasma Physics Laboratory Publications and Reports web site in Fiscal Year 2004. The home page for PPPL Reports and Publications is: http://www.pppl.gov/pub_report/

DOE and DOE Contractors can obtain copies of this report from:

U.S. Department of Energy

Office of Scientific and Technical Information

DOE Technical Information Services (DTIS)

P.O. Box 62

Oak Ridge, TN 37831

Telephone: (865) 576-8401

Fax: (865) 576-5728

Email: reports@adonis.osti.gov

This report is available to the general public from:

National Technical Information Service

U.S. Department of Commerce

5285 Port Royal Road

Springfield, VA 22161

Telephone: $1-800-553-6847$ or

(703) $605-6000$

Fax: (703) 321-8547

Internet: http://www.ntis.gov/ordering.htm 


\title{
Measurement of the Internal Magnetic Field of Plasmas using an Alpha Particle Source
}

\author{
S.J. Zweben, D.S. Darrow, P.W. Ross \\ Princeton Plasma Physics Laboratory, Princeton, NJ 08540
}

J.L. Lowrance, G. Renda

Princeton Scientific Instruments, Inc., Monmouth Junction, NJ 08852

\begin{abstract}
The internal magnetic fields of plasmas can be measured under certain conditions from the integrated $\mathrm{v}$ x B deflection of $\mathrm{MeV}$ alpha particles emitted by a small radioactive source. This alpha source and large-area alpha particle detector would be located inside the vacuum vessel but outside the plasma. Alphas with a typical energy of $5.5 \mathrm{MeV}\left({ }^{241} \mathrm{Am}\right)$ can reach the center of almost all laboratory plasmas and magnetic fusion devices, so this method can potentially determine the $\mathrm{q}(\mathrm{r})$ profile of tokamaks or STs. Orbit calculations, background evaluations, and conceptual designs for such a vxB (or "AVB") detector are described.
\end{abstract}




\section{Introduction}

The internal magnetic fields of fusion plasmas have been measured through the Motional Stark Effect [1], the Zeeman effect [2], Faraday rotation [3-4]. These diagnostics have succeeded in measuring the poloidal magnetic field profile of tokamaks but are difficult to implement, especially on smaller devices, due to their complexity and cost.

Previously, a collimated beam of injected fast ions was used to measure the $q(r)$ profile in the ATC tokamak [5], and collimated detectors were proposed to measure the $\mathrm{q}(\mathrm{r})$ profile using $3 \mathrm{MeV}$ proton emission [6]. Heavy ion beam probes (HIBP) have also been considered for internal plasma magnetic field measurements; for example, the light emission from a HIBP can be used to map out the beam trajectory inside the plasma, and thus measure the vxB deflection and local B field seen by the beam [7]. Another recent proposal is to use a high-powered short-pulse laser to generate $\approx 100 \mathrm{keV}$ electrons inside the plasma [8], which would then follow the magnetic field line to the wall, such that the wall x-ray burst could help reconstruct internal magnetic field to some extent.

This paper describes the potential use of an alpha particle source to measure the internal magnetic structure of fusion plasmas. This source would most likely be ${ }^{241} \mathrm{Am}$ with a strength $\approx 1 \mathrm{mCi}$ and an alpha energy of $\approx 5.5 \mathrm{MeV}$. The gyroorbits of these alphas can reach the center of a magnetized plasma of magnetic field " $\mathrm{B}$ " and radius "a" roughly whenever $\mathrm{B}(\mathrm{kG}) \bullet \mathrm{a}$ (meters) $\leq 3$. This is condition is marginally satisfied for NSTX at PPPL where $\mathrm{B} \approx 4.5 \mathrm{kG}, \mathrm{a} \approx 0.65 \mathrm{~m}$, as shown below. It is easily satisfied for most smaller magnetic fusion devices and laboratory plasmas; however, it is not satisfied on large fusion devices such as JET, which are designed to confine $3.5 \mathrm{MeV}$ alpha particles.

\section{Alpha particle orbit calculations}

The AVB alpha particle source would be located inside the vacuum vessel but well outside the plasma, as illustrated in Fig. 1. The alphas would first pass through a highly 
collimated aperture to fix their initial direction, and then travel through the plasma and back out again while integrating the local $\mathrm{vxB}$ force. Their impact location would be measured by a large area detector at the vessel wall.

\section{A. Simplified alpha orbit calculations with constant B}

Since the actual alpha particle orbits in a realistic 3-D magnetic geometry are generally complicated and difficult to visualize, the principles of this measurement will first be explained with a few simplified examples. These examples are chosen for a plasma of the approximate size and B field of the National Spherical Torus Experiment (NSTX) at PPPL. More realistic orbit calculations for NSTX are in Sec. II.B and II.C.

The simplest possible AVB measurement would be of the magnitude of a uniform magnetic field in a known direction by a single monoenergetic alpha particle source. This case would be relevant for plasmas which have largely self-generated internal magnetic fields, such as an FRC or a high- $\beta$ ST. For the simplified calculations, as illustrated in Fig. 2, an alpha particle source is assumed to be at $\mathrm{x}=50 \mathrm{~cm}, \mathrm{y}=-50 \mathrm{~cm}$, and $\mathrm{z}=0$, where $\mathrm{x}$ corresponds to the radial direction, $\mathrm{y}$ corresponds to the vertical direction, and $\mathrm{z}$ is the magnetic field (i.e. toroidal) direction. The wall where the AVB detector is located is assumed to be at $\mathrm{x}=50 \mathrm{~cm}$ and extends in the $\mathrm{y}$ - and z-directions.

At the top of Fig. 2 is shown a "baseline" case (solid line) for an alpha particle of energy $E=5 \mathrm{MeV}$ which is launched at angle of $\theta=0^{\circ}$ in the $(x, y)$ plane perpendicular to a spatially uniform magnetic field $\mathrm{B}_{\mathrm{Z}}=6.5 \mathrm{kG}$. This orbit is a simple circle with a gyroradius $\rho=50 \mathrm{~cm}$ with no motion along the $\mathrm{z}$-direction. Also shown at the top of Fig. 2 are two other cases with $B_{z}=5.5$ and $7.5 \mathrm{kG}$, both for the same $\theta=0^{\circ}$. The variation in the impact position in the $\mathrm{y}$-direction for a given variation in magnetic field $\Delta \mathrm{B}$ is roughly:

$$
\Delta \mathrm{y}(\mathrm{B}) \approx 2 \rho \cdot \Delta \mathrm{B} / \mathrm{B} \cdot \mathrm{f}
$$


Here " $\mathrm{f}$ " is an "orbit completion factor", where $\mathrm{f}=1$ for the half-completed orbit of Fig. 2(a) where $\theta=0^{\circ}$, and $\Delta \mathrm{y}(\mathrm{B}) \approx \pm 13-20 \mathrm{~cm}$ for $\Delta \mathrm{B}=0.1 \mathrm{kG}$. For other cases $\mathrm{f} \leq 1$, e.g. for $\approx 1 / 3$ completed orbits with $\theta= \pm 30^{\circ}, \mathrm{f} \approx 0.8$.

A second simple example is shown at the bottom of Fig. 2, in which the magnetic field is fixed at $B_{z}=6.5 \mathrm{G}$ and the alpha energy is fixed at $E=5 \mathrm{MeV}$, but the initial alpha launch angle is varied between $\theta=0^{\circ}, 10^{\circ}$ and $20^{\circ}$. This type of alpha impact variation would be obtained for a finite alpha source aperture in the y-direction. For orbits near $\theta=0^{\circ}$ this "instrumental" variation $\Delta y(\theta)$ is relatively small, since these orbits have a natural "focus" when the orbit is exactly half-completed, as in a magnetic spectrometer. For finite $\theta<30^{\circ}$ or so, this variation is approximately (from the height of a circle rotated about a point):

$$
\Delta y(\theta) \approx 2 \rho \cdot \sin \theta \cdot \Delta \theta \text { (radians) }
$$

The alpha impact positions vary by about $\Delta y \approx \pm 4-8 \mathrm{~cm}$ for variations of $\Delta \theta \approx \pm 10^{\circ}$ around $\theta=20^{\circ}$.

The implication of Eqs. 1 and 2 is that the angular spread in the collimation of the alpha source $\Delta \theta$ in the $\mathrm{y}$-direction determines the accuracy with which the magnitude of $\mathrm{B}$ can be measured in situations like this. When the instrumental variation $\Delta y(\theta)$ is comparable to the magnetic field induced variation $\Delta y(B)$, the $\Delta \mathrm{B}$ which can be resolved for a given $\Delta \theta$ is approximately (from Eq. 3 and 4):

$$
\Delta \mathrm{B} / \mathrm{B} \approx \sin \theta \cdot \Delta \theta \text { (radians) } / \mathrm{f}
$$

For the cases of Fig. 2(b) with $\theta \approx 20^{\circ}, \Delta \theta \approx 10^{\circ}$ and $\mathrm{f} \approx 0.9$, the magnitude of $\mathrm{B}$ can be determined to within $\Delta \mathrm{B} / \mathrm{B} \approx 6 \%$, assuming a perfectly resolving detector in the y-direction. 
Obviously, there is a similar but simpler relationship for the minimum $\Delta \mathrm{B}$ which can be measured given an uncertainty in the alpha source energy $\Delta \mathrm{E}$ (which was assumed to be zero in the estimates above); namely:

$$
\Delta \mathrm{B} / \mathrm{B} \approx 1 / 2(\Delta \mathrm{E} / \mathrm{E})
$$

However, the alpha source will in most cases have a narrow energy spectrum, e.g. ${ }^{241} \mathrm{Am}$ can have $\Delta \mathrm{E} / \mathrm{E}<0.01$, so this uncertainty due to the alpha energy in should be relatively small in such cases.

In plasma devices such as a low beta ST, tokamak or stellarator the main unknown is in the direction of the magnetic field, which is commonly described by the $q(\mathrm{r})$ profile, rather than its magnitude (which is nearly constant for low $\beta$ ). A simple example of the effect of changing the direction of $\mathrm{B}$ for a fixed magnitude of $\mathrm{B}$ is shown at the top of Fig. 3. Here again there are three cases: the solid line correspond to the same baseline case of Fig. 2, with $\mathrm{B}_{\mathrm{z}}=6.5 \mathrm{~T}$, and alpha particle energy of $\mathrm{E}=5 \mathrm{MeV}$ started at an angle of $\theta=0^{\circ}$, and a vertical field $\mathrm{B}_{\mathrm{y}}=0$. The dotted and dashed lines show alpha orbits with the same alpha parameters and magnetic field magnitude, but with differing magnetic field line angles $\chi$, i.e. $\mathrm{B}_{\mathrm{y}}=6.5 \cdot \sin \chi$ and $\mathrm{B}_{\mathrm{z}}=6.5 \cdot \cos \chi$.

From Fig. 3 it can be seen that the y-impact position is only slightly affected by the direction of the magnetic field; however, the z-impact position is significantly changed for $\chi$ $\neq 0$. In these cases the initial alpha direction is still perpendicular to $B$, so the alpha trajectories are just circles which are rotated by the angle $\chi$ about their initial alpha direction. For moderately small $\chi<30^{\circ}$, the projection of these circles on the $(\mathrm{x}-\mathrm{z})$ plane are ellipses which have a $\mathrm{z}$-impact position of $\mathrm{z}(\chi) \approx 2 \rho \bullet \sin \chi$, which varies as:

$$
\Delta \mathrm{z}(\chi) \approx 2 \rho \cdot \cos \chi \cdot \Delta \chi(\text { radians })
$$

The alpha impact positions for the cases of Fig. 3 vary by $\Delta \mathrm{z} \approx \pm 17 \mathrm{~cm}$ for variations of $\Delta \chi \approx$ $\pm 10^{\circ}$ around $\chi=10^{\circ}$. 
Finally, the effect of a finite variation in the toroidal launch angle in the z-direction is shown at the bottom of Fig. 3, where this angle is varied between $\varphi=10^{\circ}, 20^{\circ}$ and $30^{\circ}$. This type of alpha impact variation would be obtained for a finite alpha source aperture in the zdirection. Again, there is relatively little variation of the alpha impact in the y-direction, but in this case the alpha orbits are helices which have a z-impact position $\mathrm{z}(\varphi) \approx \pi \rho \bullet \sin \varphi$, which for relatively small varies $\varphi<30^{\circ}$ varies as:

$$
\Delta \mathrm{z}(\varphi) \approx \pi \rho \cdot \cos \varphi \bullet \Delta \varphi(\text { radians })
$$

The alpha impact positions vary by $\Delta \mathrm{z} \approx \pm 25 \mathrm{~cm}$ for variations of $\Delta \varphi \approx \pm 10^{\circ}$ around $\varphi=20^{\circ}$.

The implication of Eqs. 5 and 6 is that the angular spread in the collimation of the alpha source $\Delta \varphi$ in the z-direction determines the accuracy with which the direction of B can be measured in situations like this. When the instrumental variation $\Delta z(\varphi)$ is comparable to the magnetic field direction-induced variation $\Delta z(\chi)$ for alpha orbits with relatively small $\theta, \varphi, \chi$, the uncertainly in the magnetic field line angle for a given $\Delta \varphi$ is approximately (from Eq. 3 and 4):

$$
\Delta \chi \approx(\pi / 2) \Delta \varphi
$$

Thus for the case of Fig. 3(a) with $\chi \approx 20^{\circ}$, the direction of B can be determined to within $\Delta \chi \approx$ $5^{\circ}$ with an alpha source aperture angle of $\Delta \varphi \approx 3^{\circ}$, assuming a perfectly resolving detector in the z-direction.

Several tentative conclusions can be drawn from these simple examples:

a) both the magnitude and direction of a uniform B field can be determined by the impact positions of alpha particles launched approximately perpendicular to B, 
b) the uncertainty in the magnitude and/or direction of $\mathrm{B}$ in these cases is determined by the angular spread in the alpha source direction both across and along B,

c) the simplest alpha trajectory for measuring these magnetic fields is probably a semicircle, the radius of which can be used to determine the magnitude of $\mathrm{B}$ and the angle of which can be used to determine the direction of $\mathrm{B}$,

\section{II.B Cylindrical model alpha orbit calculations}

Sample results from a cylindrical calculation of alpha particle orbits with a varying $\mathrm{q}(\mathrm{r})$ profile are shown in Fig. 4. The magnetic field model is a approximation of NSTX in which the toroidal magnetic field is taken to be constant $\mathrm{B}=5 \mathrm{kG}$ and the poloidal field profile is specified by $\mathrm{q}(\mathrm{r})$, where $\mathrm{q}(0)$ is varied from 0.5 to 4 , and the edge $\mathrm{q}(\mathrm{a})=1$ in all cases. The radially averaged magnetic field line angles for this range of $\mathrm{q}(\mathrm{r})$ vary from $\chi \approx 17^{\circ}$ for the $\mathrm{q}(0)=0.5$ case to $\chi \approx 8^{\circ}$ for the $\mathrm{q}(0)=4$ case.

At the top of Fig. 4 are typical alpha orbits launched at a poloidal angle $\theta=20^{\circ}$ and a toroidal angle $\varphi=0^{\circ}$ for these various $q(0)$ values. As explained in Sec. II.B, the direction of the magnetic field, as parameterized here by $\mathrm{q}(0)$, does not significantly change the alpha orbits in the radial vs. poloidal plane, but does significantly change the alpha impact location in the toroidal direction due to the tilt of the alpha orbit plane at the magnetic field line angle $\chi$. Here this variation is $\Delta \mathrm{z}(\chi) \approx 15 \mathrm{~cm}$ for this $\Delta \chi \approx 9^{\circ}$, which is roughly consistent with Eq. 5 for $\rho \approx 50 \mathrm{~cm}$.

At the bottom of Fig. 4 are the detector (i.e. wall) impact locations for various $q(0)$ and poloidal launch angles $\theta$, where the 5 points plotted for each case correspond to the impacts of the orbits passing through the centroid and the corners of the alpha source aperture. Here the aperture angles were chosen so that the impact locations at various $q(r)$ and $\theta$ were separated in the detector plane, i.e. $\Delta \theta=2^{\circ}$ in the poloidal direction and $\Delta \varphi=0.4^{\circ}$ in the toroidal direction. The toroidal variation in the detector impact position due to the 
variation is toroidal launch angle is $\Delta \mathrm{z}(\varphi) \approx 1 \mathrm{~cm}$, which is roughly consistent with Eq. 6 for $\rho \approx 50 \mathrm{~cm}$.

The tentative conclusion to be drawn from Fig. 4 is that the $q(r)$ profiles chosen for this model could potentially be distinguished by the AVB technique with a $5 \mathrm{MeV}$ alpha source having a toroidal aperture angle of $\Delta \varphi \approx 0.4^{\circ}$. This relatively small aperture angle will reduce the alpha flux considerably, as discussed in Sec. III.

\section{II.C Realistic alpha orbit calculations for NSTX}

Alpha particle orbits for a realistic NSTX plasma case are illustrated in Figs. 5. This discharge (\#104033) had $\mathrm{B}=4.5 \mathrm{kG}$ and $\mathrm{I}=770 \pm 10 \mathrm{kA}$, and the two $\mathrm{q}(\mathrm{r})$ profiles shown had $\mathrm{q}(0) \approx 1.0$ (solid lines) or $\mathrm{q}(0) \approx 1.7$ (dashed lines) within $\mathrm{r} / \mathrm{a} \approx 0.5$, both with the same $\mathrm{q}(\mathrm{a}) \approx$ 8 (according to EFIT). Here the alpha source energy was $5.5 \mathrm{MeV}$ and three alpha particle orbits are plotted for each of two different q(r) profiles. These three orbits have poloidal launch angles of $\theta=0^{\circ}, 10^{\circ}$ and $20^{\circ}$ and a toroidal launch angle of $\varphi=28^{\circ}$ (i.e. nearly radial).

This difference in $\mathrm{q}(\mathrm{r})$ profiles did not significantly affect the orbits in the poloidal plane (left), but there was a clear difference in the toroidal impact location between the two $\mathrm{q}(\mathrm{r})$ profiles (right). This is very similar to the results of Sec. II.B.

Fig. 6 shows a plot of the alpha particle wall impact location for the orbits of Fig. 5, along with the locations at 8 points around the edge of an assumed source aperture for each. The aperture angles for Fig. 6 were chosen so that the range of toroidal impact location due to the finite aperture size would be significantly less than the difference in toroidal impact locations between the two $\mathrm{q}\left(\mathrm{r}\right.$ ) profiles. For this case these angles were $\Delta \theta=5^{\circ}$ (in the poloidal direction) and $\Delta \varphi=0.6^{\circ}$ (in the toroidal direction). A measurement with these apertures could potentially distinguish these $q(r)$ profiles. 
Since the alpha impact locations for various $\mathrm{q}(\mathrm{r})$ profiles form smooth curves when the poloidal launch angle is varied, a source aperture with a continuous slit in the poloidal direction would allow increased signal without significantly degrading the observable variation due to $\mathrm{q}(\mathrm{r})$.

The requirement for spatial resolution of $\mathrm{B}$ inside the plasma implies a need for multiple alpha source apertures each defining a distinct alpha orbit trajectory, e,g, with varying initial alpha angles $\theta$ and $\varphi$, and/or alpha energy E. A single large area AVB detector could probably identify the impact locations for $\approx 10$ source apertures. Thus it seems possible to measure up to $\approx 10$ spatially resolved points on the $\mathrm{q}(\mathrm{r})$ profile with a single AVB detector.

\section{Alpha detector design}

The AVB alpha detector would be a 2-D position sensitive detector with sufficient spatial resolution to localize the alpha impact locations from an alpha source with $\approx 10$ apertures. For a conceptual design we assume an ${ }^{241} \mathrm{Am}$ source of $\approx 5.5 \pm 0.1 \mathrm{MeV}$ alphas with a strength of $\approx 1 \mathrm{mCi}$, or $3.7 \times 10^{7}$ alphas/sec (e.g. from Isotope Products, Inc). The alpha flux through a single aperture slit with $\Delta \theta=10^{\circ}$ (in the poloidal direction) and $\Delta \varphi=0.6^{\circ}$ (in the toroidal direction) would be $\approx 10^{4}$ alphas/sec. To localize the alpha impacts from a single aperture would require $\approx 100$ counts, thus a typical measurement timescale would be $\approx 10$ msec.

The required detector spatial resolution (i.e. pixel size) is determined by the desired $\mathrm{q}(\mathrm{r})$ profile resolution. For the NSTX case of Fig. 2, the two $\mathrm{q}(\mathrm{r})$ profiles are separated by $\approx$ $5 \mathrm{~cm}$ at the detector, so a spatial resolution of $\approx 1 \mathrm{~cm}$ would be sufficient. The size of the AVB detector is determined by the range of $\mathrm{q}(\mathrm{r})$ and $\mathrm{B}$ field variations to be measured in a given device. For the NSTX cases of Fig. 2 the detector should have a size of $\approx 40 \mathrm{~cm} \mathrm{x} 40$ $\mathrm{cm}$. 
These AVB source and detector requirements for NSTX are summarized in Table 1. Assuming a design with 10 apertures as described above, the total alpha count rate would be $\approx 10^{5}$ alphas/sec. For other devices the required detector resolution and size will scale roughly linearly with the alpha gyroradius. As discussed below, background discrimination may require a modest amount of alpha energy resolution from this detector.

The main background in deuterium fusion plasmas such as NSTX would the escaping flux of D-D fusion products from the plasma, including the $1 \mathrm{MeV}$ triton, the $3 \mathrm{MeV}$ proton, and the $0.8 \mathrm{MeV}^{3} \mathrm{He}$ from D-D reactions (the D-T and $\mathrm{D}-{ }^{3} \mathrm{He}$ burn-up products should be negligible for plasmas of interest). The $1 \mathrm{MeV}$ triton and $0.8 \mathrm{MeV}^{3} \mathrm{He}$ can be blocked by a $5 \mu \mathrm{m}$ gold foil covering the detector, after which the $5.5 \mathrm{MeV}$ alpha will still have $\approx 3 \mathrm{MeV}$ [9]. However, the range of the $3 \mathrm{MeV}$ proton in gold is $\approx 30 \mu \mathrm{m}$ so this gold layer will not stop the proton. Since the gyroradius of the $3 \mathrm{MeV}$ proton is nearly the same as a $5 \mathrm{MeV}$ alpha particle, a large fraction of D-D protons reach to the wall whenever the AVB alpha particle can reach the plasma center. Therefore the escaping $3 \mathrm{MeV}$ proton flux can be a significant background whenever the D-D fusion rates becomes very much larger than the AVB alpha source rate.

Estimates of the ratio of the $3 \mathrm{MeV}$ proton rate to the AVB alpha rate for three plasma devices is given in Table 2, assuming an AVB detector design similar to that of Table 1. The proton background should be negligible for low temperature (or hydrogen fueled) devices like the Electric Tokamak (ET). However, for higher temperature devices like MST or NSTX this background will make the AVB measurement more challenging. One detection strategy is to discriminate the AVB alphas from the protons through pulse height analysis; for example, in a silicon detector just thick enough to stop a $3 \mathrm{MeV}$ AVB alpha $(\approx 12 \mu \mathrm{m})$ the $3 \mathrm{MeV}$ proton will deposit $\leq 0.3 \mathrm{MeV}$. Thus the pulse height discrimination of the alphas from the protons should relatively easily, at least when the proton count rate is $\leq$ 10 times the alpha count rate. 
The only other significant background should be due to x-rays emitted from the plasma. However, a gold coating or foil over the detector would block most of the plasma xrays (along with visible and UV light); for example, the fraction of thermal plasma x-ray energy passing through a $5 \mu \mathrm{m}$ gold foil would be $\approx 10^{-6}$ for $\mathrm{T}_{\mathrm{e}}=0.5 \mathrm{keV}, 10^{-4}$ for $\mathrm{T}_{\mathrm{e}}=1$ $\mathrm{keV}$, and $\approx 10^{-2}$ for $\mathrm{T}_{\mathrm{e}}=2 \mathrm{keV}$ [9]. Thus the $\mathrm{x}$-ray background for the devices of Table 2 would be negligible for ET, moderate for MST and Ohmic NSTX plasmas, but large for NBI heated NSTX plasmas. However, this X-ray background will consist of a very large number of very small pulses $(\leq 1 \mathrm{keV})$, so a good pulse height discrimination system might be able to eliminate most of this x-ray background.

Turning to detector implementation, the alpha detection requirements of Table 1 can certainly be met by an array of discrete silicon diode detectors (e.g. ORTEC Ultra series). However, a 40x40 array such detectors would be prohibitively expensive and complicated to monitor.

A simpler technique would be to use an optically coupled scintillator screen. It is well known that thin powdered phosphors such as $\mathrm{ZnS}$ can detect single alpha particles, and screens of 40 x $40 \mathrm{~cm}$ can be easily made. The crystal size of these phosphors is typically only a few $\mu \mathrm{m}$, so the alpha energy resolution will be poor; however, if the backgrounds are relatively small this should be acceptable. The detector spatial resolution can be $\approx 0.1 \mathrm{~cm}$, and the response time (depending on phosphor type) can be $\approx 1-30 \mu \mathrm{sec}$. The screen can be view from behind by a single large lens, or if space is limited, by an array of fiber optically coupled "funnel/cones" similar to that employed in Cerenkov radiation detectors. Most likely thousands of single alphas impacts can be seen on such a screen using an intensified video camera with a nominal integration time of $16 \mathrm{msec} /$ frame.

Another possible detection technique would be a large-area position sensitive amorphous silicon detector. For example, $40 \mathrm{~cm}$ x $40 \mathrm{~cm}$ amorphous silicon diode arrays with 200 micron spatial resolution are already used for industrial and medical x-ray imaging applications (e.g. from Perkin Elmer). There is also ongoing work to develop large area amorphous silicon detector arrays for particle detection in physics experiments [10]. An 
amorphous silicon detector array should have much better energy resolution than a phosphor screen; however, signal handling at a total count rate of $\approx 100 \mathrm{kHz}$ may be difficult.

\section{Other Applications}

Although the backgrounds are high when $\mathrm{T}_{\mathrm{e}}(0)>1 \mathrm{keV}$, this diagnostic might be able to make an edge $\mathrm{q}(\mathrm{r})$ measurement by using movable baffles to block the $\mathrm{x}$-rays and $3 \mathrm{MeV}$ protons from the plasma center. In this case the detector would see only alphas passing through the edge and incident onto the detector at a small angle, along with the local backgrounds from this edge region. The AVB diagnostic could also be used to measure the magnitude of the internal B in "self-organized" plasmas like FRCs or high $\beta$ STs, or to map the 3-D magnetic field structure in stellarators. Finally, if alpha sources can be found with a strength of $\approx 1 \mathrm{Ci}$ (instead of $1 \mathrm{mCi}$ ), it might be possible to measure time-dependent internal magnetic field fluctuations this way.

Acknowledgments: We thank D. Johnson and P. Efthimion of PPPL for supporting this study, S. Kaye for information about NSTX neutron rates, and P. Schoch for discussions about the heavy ion beam probe. This work was performed under DOE contract \# DEAC02-76CHO3073. 


\section{References:}

[1] S. Reyes Cortes et al, Rev. Sci. Inst. 74, 1596 (2003)

[2] D.M. Thomas, Rev. Sci. Inst. 74, 1541 (2003)

[3] D.L. Brower et al, Rev. Sci. Inst. 74, 1534 (2003)

[4] K.C. Lee et al, Rev. Sci. Inst. 74, 1621 (2003)

[5] R.J. Goldston, Phys. Fluids 21, 2346 (1978)

[6] W.W. Heidbrink et al, Rev. Sci. Inst. 56, 501 (1985)

[7] D.R. Demers et al, Rev. Sci. Inst. 74, 2103 (2003)

[8] H.S. McLean et al, Rev. Sci. Inst. 74, 1547 (2003)

[9] NIST: http://physics.nist.gov/PhysRefData

[10] C. Hordequin et al, Nucl. Inst. Methods A 456, 284 (2001) 


\section{Table 1: Alpha source and detector requirements}

Alpha source energy: $\approx 5.5 \pm 0.1 \mathrm{MeV}$

Alpha source strength: $\approx 1 \mathrm{mCi}\left(3.7 \times 10^{7} / \mathrm{sec}\right)$

Alpha detector pixel size: $\approx 1 \mathrm{~cm}$

Alpha detector size: $\approx 40 \mathrm{~cm} \mathrm{x} 40 \mathrm{~cm}$

Alpha detector energy resolution: $\approx 50 \%$

Alpha particle count rate $\approx 10^{4} / \mathrm{sec} /$ aperture

Table 2: AVB detector backgrounds for three devices

$\begin{array}{llll}\text { Parameter } & \text { ET }(\text { UCLA) } & \text { MST(Wisc. }) & \text { NSTX }(\text { PPPL }) \\ \mathrm{T}_{\mathrm{e}}(\mathrm{keV}) & \approx 0.5 & \approx 0.5 & \approx 0.5(\mathrm{OH})-2(\mathrm{NBI}) \\ \mathrm{n}_{\mathrm{e}}\left(10^{13} \mathrm{~cm}^{-3}\right) & \approx 0.2 & 1 & 1(\mathrm{OH})-3(\mathrm{NBI}) \\ \text { neutrons/sec } & \approx 10^{6} & \approx 10^{8} & \approx 10^{9}(\mathrm{OH})-10^{14}(\mathrm{NBI}) \\ (\text { protons } / \alpha)^{*} & <0.01 & \sim 0.1-1 & \sim 1(\mathrm{OH})-10^{5}(\mathrm{NBI}) \\ (\mathrm{x}-\mathrm{ray} / \alpha)^{* *} & \sim 0.1 & \sim 1 & \sim 1(\mathrm{OH})-10^{5}(\mathrm{NBI})\end{array}$

* (number of $3 \mathrm{MeV}$ protons / number of AVB alphas) onto detector

•• (x-ray energy flux / AVB alpha energy flux) behind $5 \mu \mathrm{m}$ gold foil 


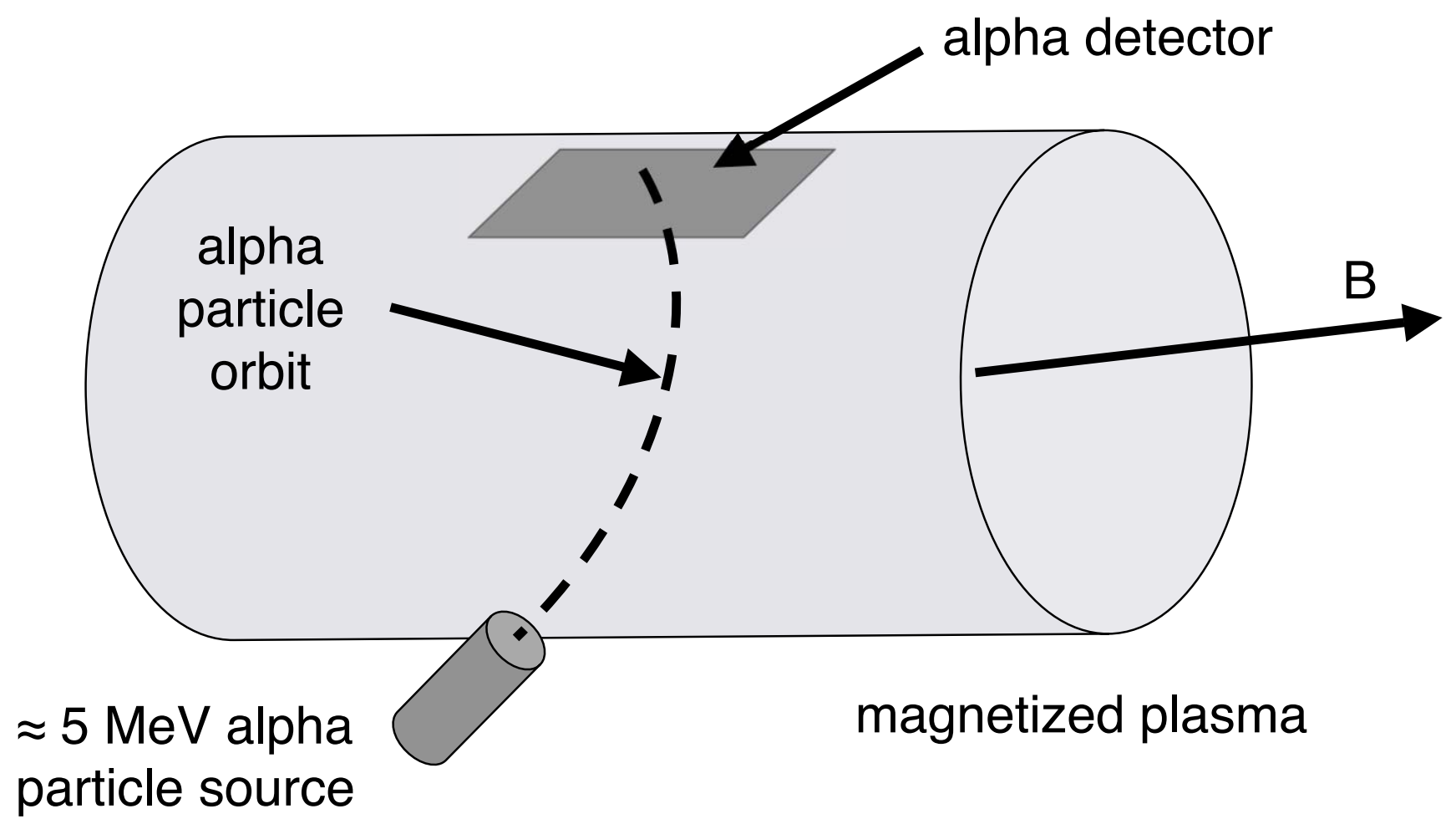

Fig. 1: Schematic illustration of the alpha vxB (AVB) detector concept. An radioactive alpha source (typically ${ }^{241} \mathrm{Am}$ ) located at the back of a collimator aims a narrow beam of alphas nearly radially into the plasma. The alpha orbits pass through the plasma, integrate the $\mathrm{vxB}$ force, and are measured at a large area detector on the wall (but well outside the plasma). 
Side View
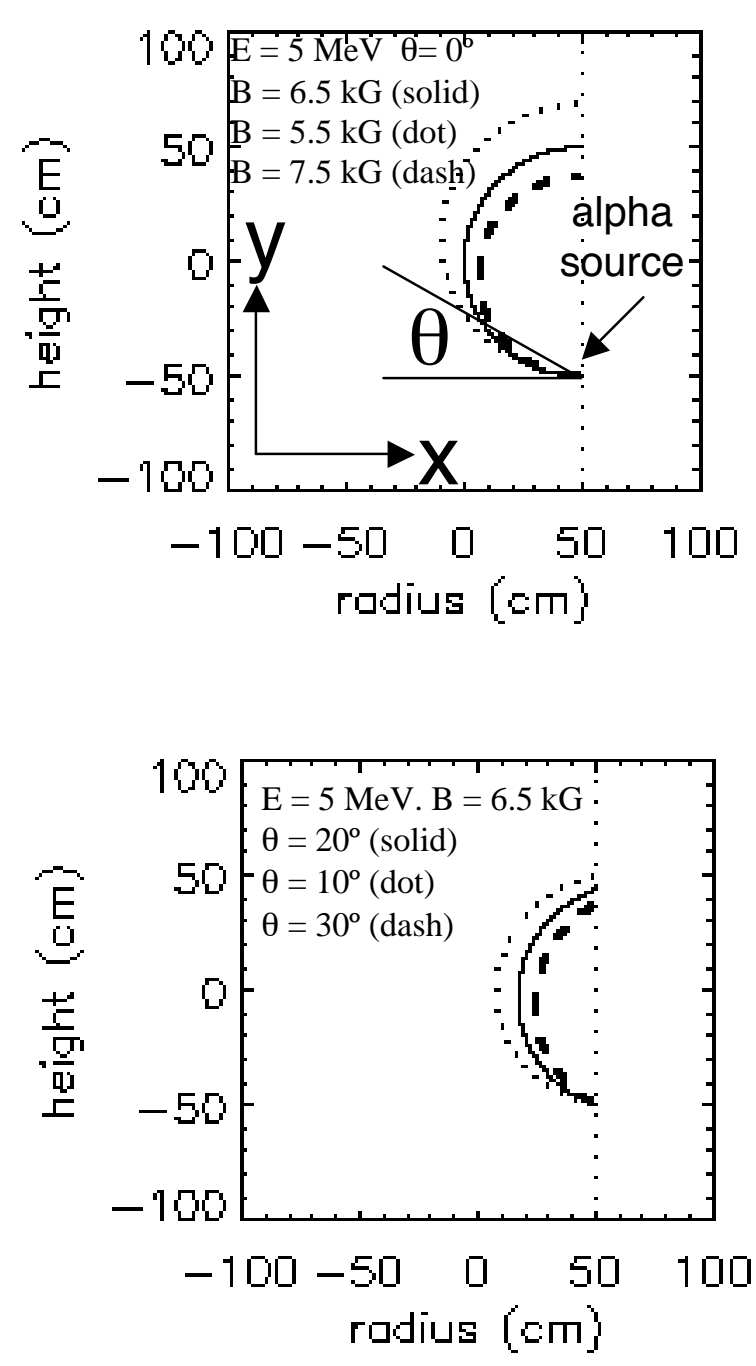

\section{Top View}
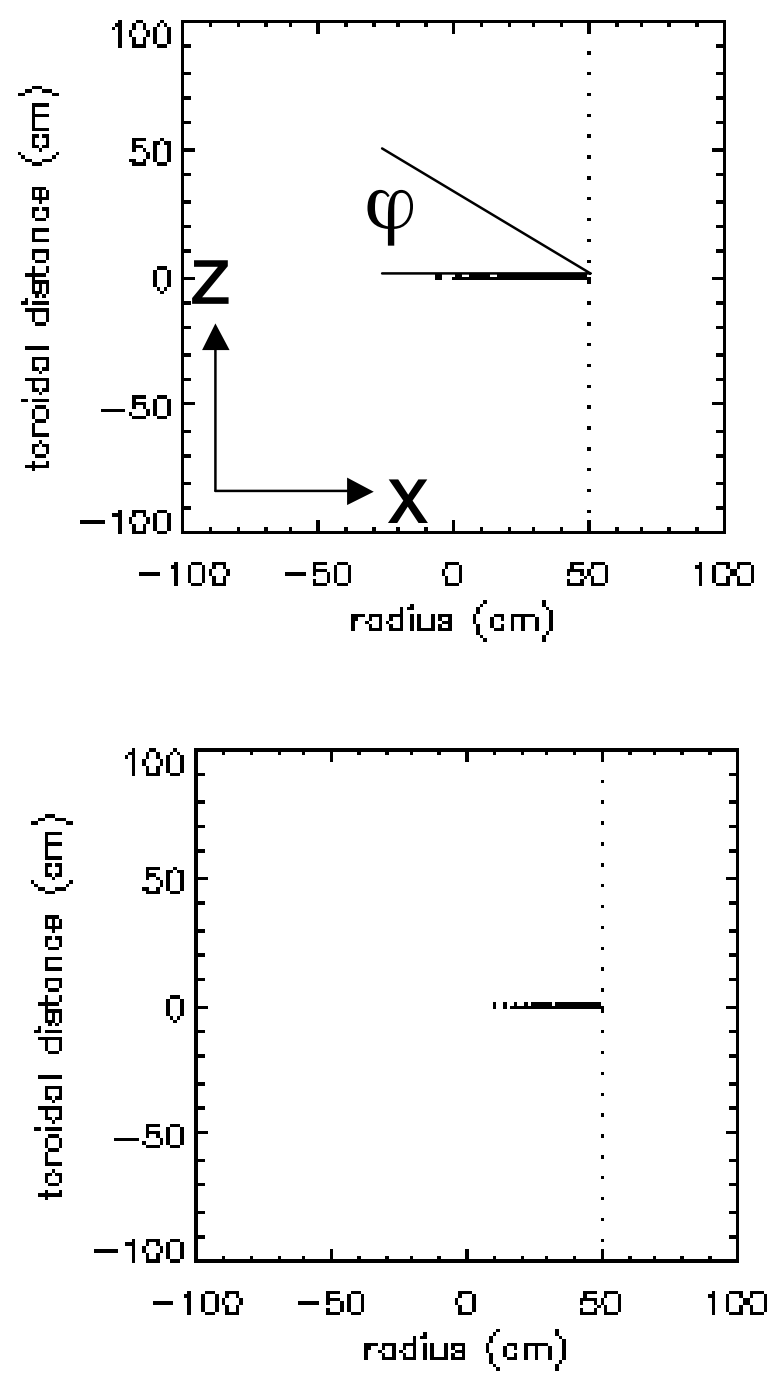

Fig. 2: Simple examples of alpha orbits with a spatially constant B field. At the left are the projections of the orbits in the $\mathrm{x}-\mathrm{y}$ plane, and at the right are the projections in the $\mathrm{x}-\mathrm{z}$ plane. At the top are three alpha orbits with $E=5 \mathrm{MeV}$ launched at a poloidal angle of $\theta=0^{\circ}$, a toroidal angle of $\varphi=0^{\circ}$, and with varying $\mathrm{B}=6.5 \mathrm{kG}$ (solid), $5.5 \mathrm{kG}$ (dot), and 7.5 $\mathrm{kG}$ (dash). At the bottom are three orbits with a constant $\mathrm{B}=6.5 \mathrm{kG}$ but a variable poloidal launch angle $\theta=20^{\circ}$ (solid), $\theta=10^{\circ}$ (dot), and $\theta=30^{\circ}$ (dash). 
Side View
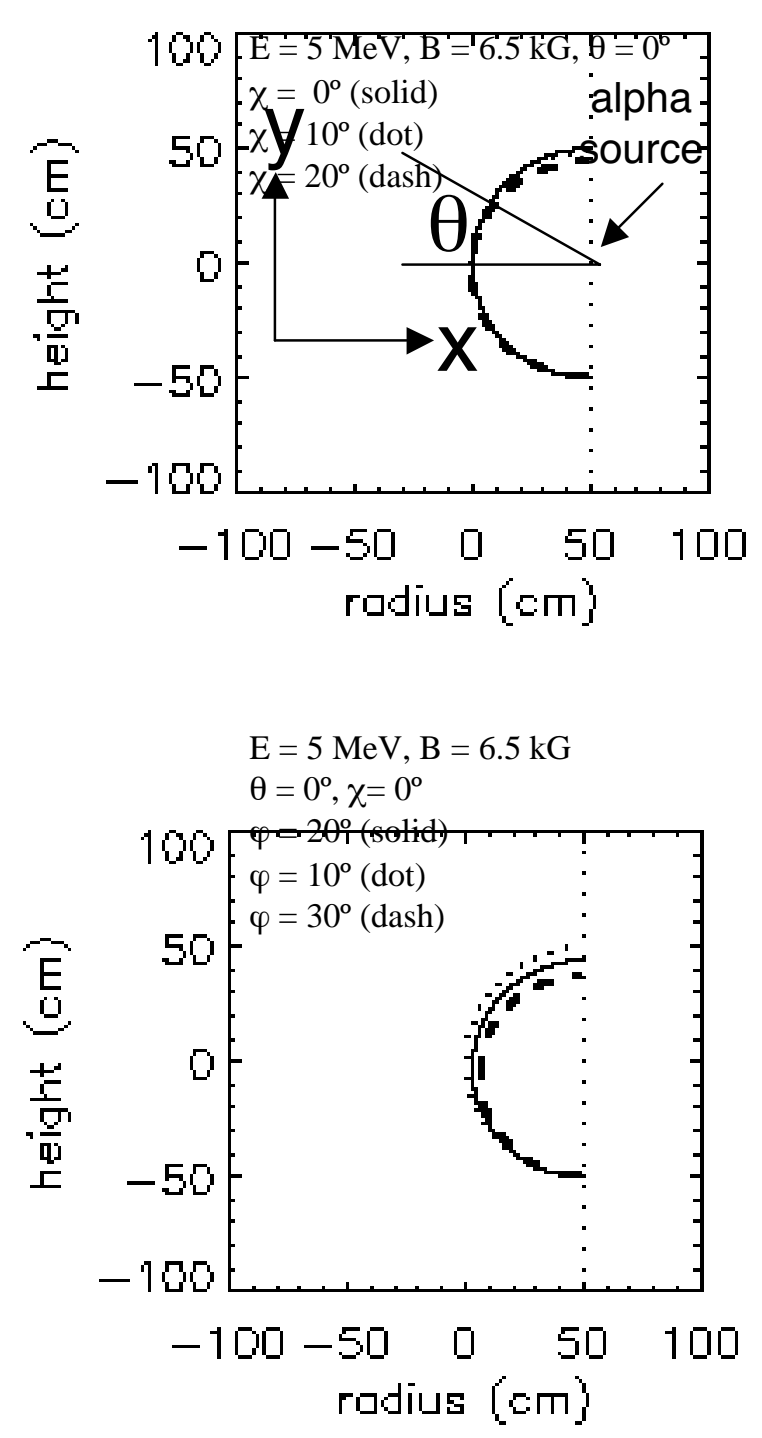

Top View
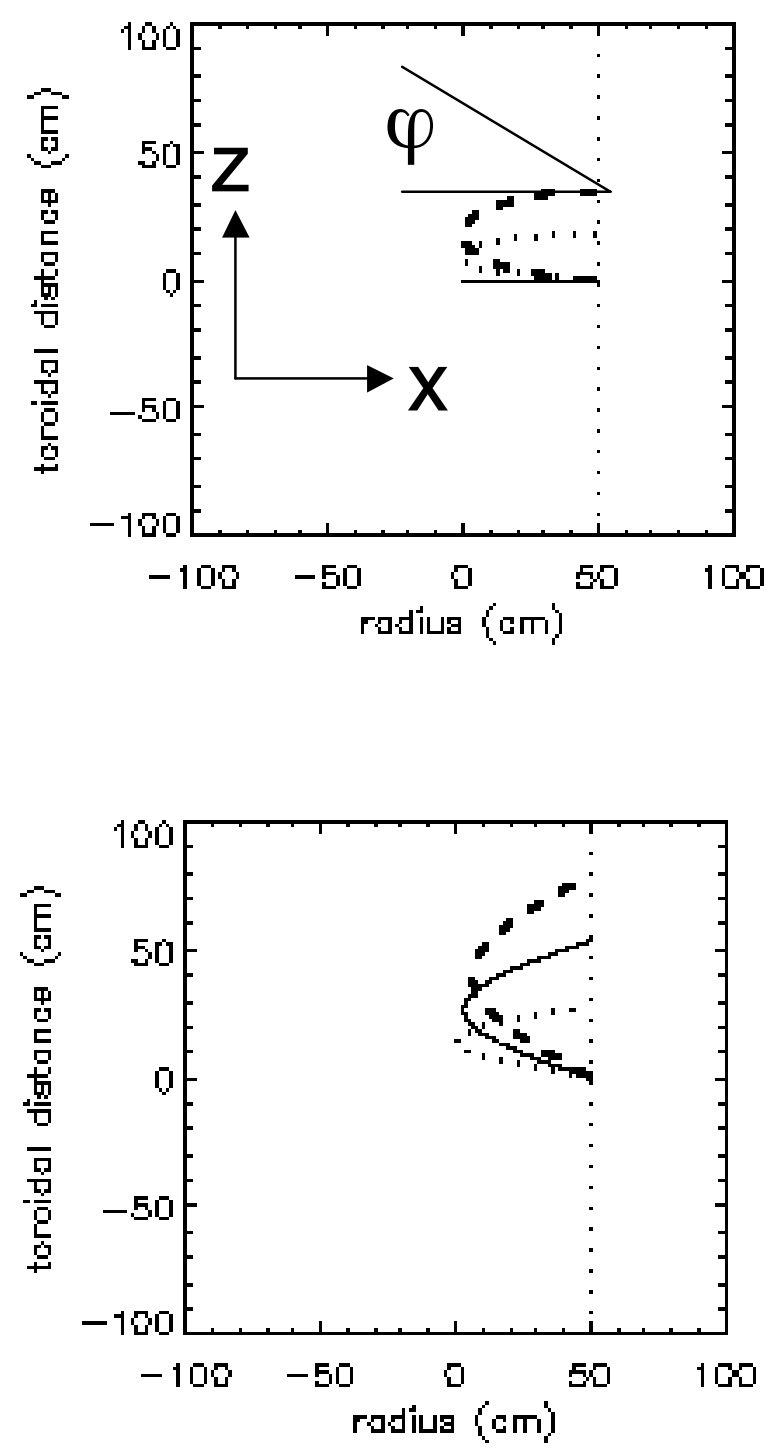

Fig. 3: Simple examples of alpha orbits with a spatially constant B field. At the left are the projections of the orbits in the $\mathrm{x}-\mathrm{y}$ plane, and at the right are the projections in the $\mathrm{x}-\mathrm{Z}$ plane. At the top are three alpha orbits with $\mathrm{E}=5 \mathrm{MeV}$ launched at a poloidal angle of $\theta=0^{\circ}$ with $B=6.5 \mathrm{kG}$, but with varying magnetic field line angles $\chi=0^{\circ}$ (solid), $\chi=10^{\circ}$ (dot), and $\chi=20^{\circ}$ (dash). At the bottom are three orbits with a constant field line angle but a variable toroidal launch angle $\varphi=20^{\circ}$ (solid), $\varphi=10^{\circ}$ (dot), and $\varphi=30^{\circ}$ (dash). 

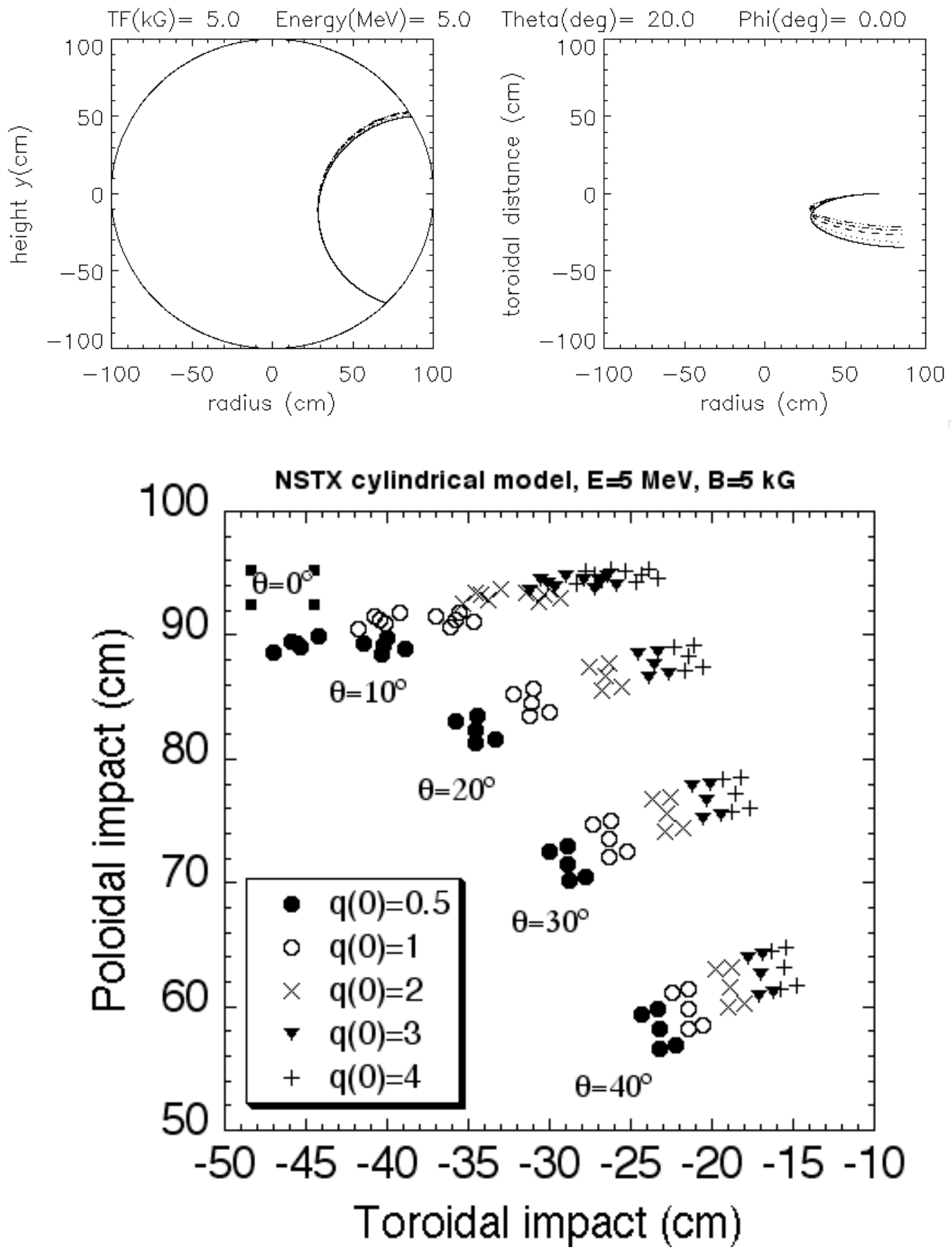

Fig. 4: Cylindrical model of AVB orbits and detector impact positions for various $q(r)$ profiles in NSTX. At the top are orbits for varying $q(0)$ with a fixed $q(a)$ with $E=5 \mathrm{MeV}$, and $\mathrm{B}=5 \mathrm{kG}$. At the bottom are the detector impact locations for varying $\mathrm{q}(0)$ and poloidal launch angle $\theta$, where the 5 points for each case correspond to the impacts of the orbits passing through the centroid and the corners of the alpha source aperture. 

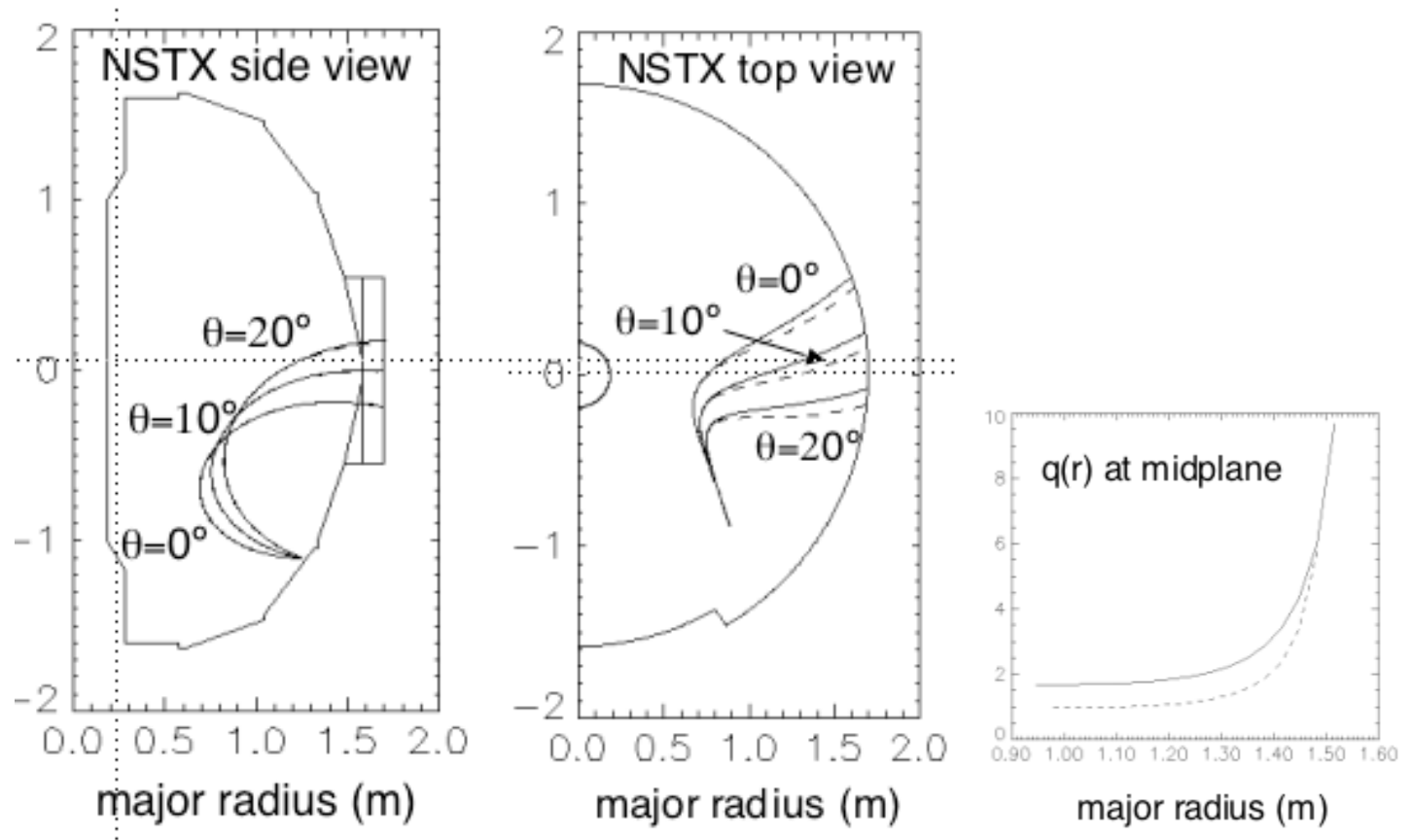

Fig. 5: Alpha particle orbit calculations for a realistic magnetic geometry in NSTX. The alphas of $5.5 \mathrm{MeV}$ are launched $\approx 45^{\circ}$ below the outer midplane at three different poloidal angles $\theta=0^{\circ}, 10^{\circ}$ and $20^{\circ}$, all with a toroidal launch angle of $\chi=28^{\circ}$ (i.e. nearly radial). The solid lines are for a $q(r)$ profile with $q(0)=1.7$ and the dashed line for $q(0)=1.0$, both with $\mathrm{q}(\mathrm{a})=8$. 


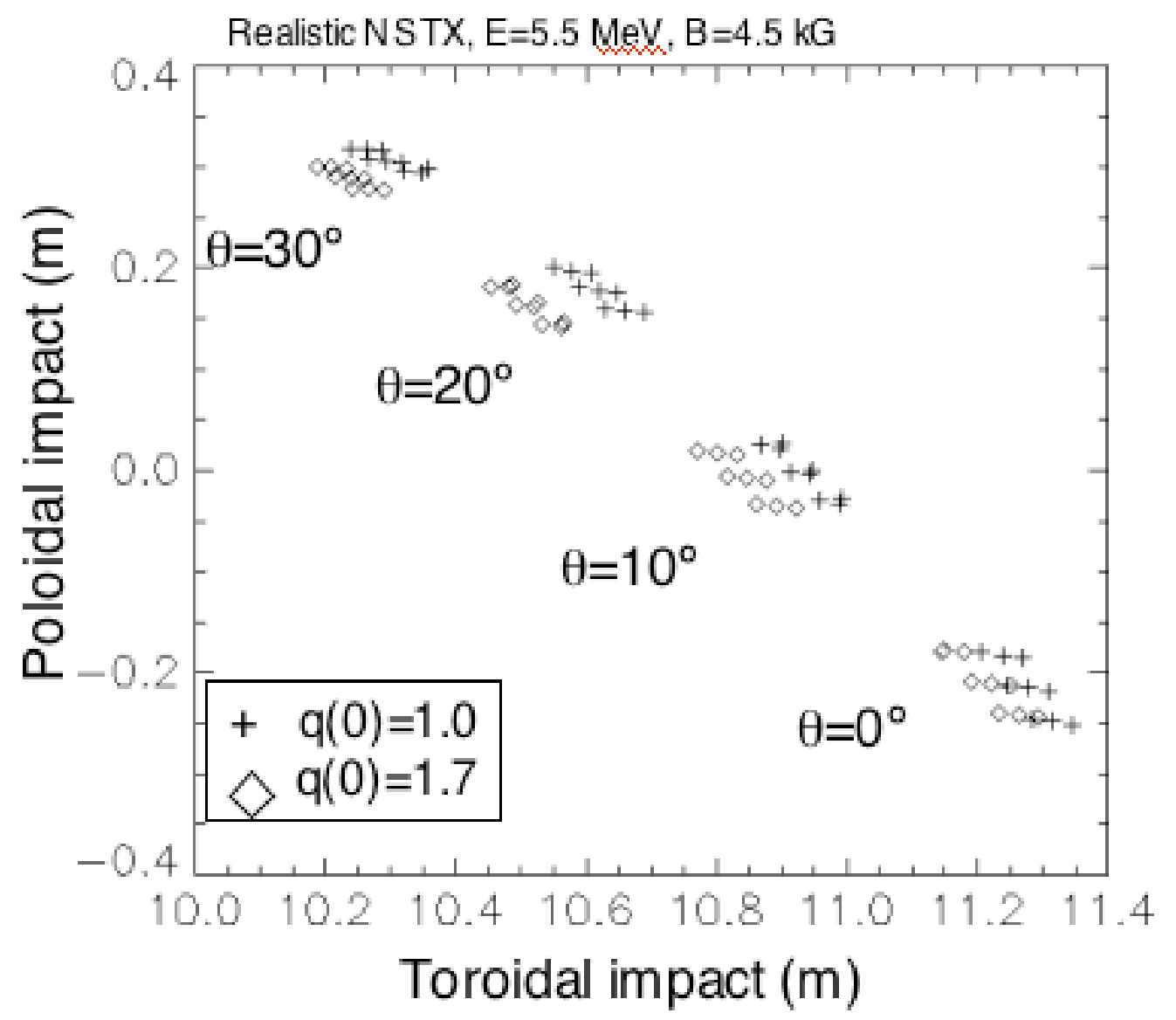

Fig. 6 Variation of the alpha particle wall impact location for the cases of Fig. 5. For each $\mathrm{q}(\mathrm{r})$ and launch angle $\theta$ there 9 points corresponding to the center and corners of the assumed alpha source aperture. For this plot, these aperture angles were $\Delta \theta=5^{\circ}$ (in the poloidal direction) and $\Delta \varphi=0.6^{\circ}$ (in the toroidal direction). 


\section{External Distribution}

Plasma Research Laboratory, Australian National University, Australia

Professor I.R. Jones, Flinders University, Australia

Professor João Canalle, Instituto de Fisica DEQ/IF - UERJ, Brazil

Mr. Gerson O. Ludwig, Instituto Nacional de Pesquisas, Brazil

Dr. P.H. Sakanaka, Instituto Fisica, Brazil

The Librarian, Culham Laboratory, England

Mrs. S.A. Hutchinson, JET Library, England

Professor M.N. Bussac, Ecole Polytechnique, France

Librarian, Max-Planck-Institut für Plasmaphysik, Germany

Jolan Moldvai, Reports Library, Hungarian Academy of Sciences, Central Research Institute for Physics, Hungary

Dr. P. Kaw, Institute for Plasma Research, India

Ms. P.J. Pathak, Librarian, Institute for Plasma Research, India

Ms. Clelia De Palo, Associazione EURATOM-ENEA, Italy

Dr. G. Grosso, Instituto di Fisica del Plasma, Italy

Librarian, Naka Fusion Research Establishment, JAERI, Japan

Library, Laboratory for Complex Energy Processes, Institute for Advanced Study, Kyoto University, Japan

Research Information Center, National Institute for Fusion Science, Japan

Dr. O. Mitarai, Kyushu Tokai University, Japan

Dr. Jiangang Li, Institute of Plasma Physics, Chinese Academy of Sciences, People's Republic of China

Professor Yuping Huo, School of Physical Science and Technology, People's Republic of China

Library, Academia Sinica, Institute of Plasma Physics, People's Republic of China

Librarian, Institute of Physics, Chinese Academy of Sciences, People's Republic of China

Dr. S. Mirnov, TRINITI, Troitsk, Russian Federation, Russia

Dr. V.S. Strelkov, Kurchatov Institute, Russian Federation, Russia

Professor Peter Lukac, Katedra Fyziky Plazmy MFF UK, Mlynska dolina F-2, Komenskeho Univerzita, SK-842 15 Bratislava, Slovakia

Dr. G.S. Lee, Korea Basic Science Institute, South Korea

Institute for Plasma Research, University of Maryland, USA

Librarian, Fusion Energy Division, Oak Ridge National Laboratory, USA

Librarian, Institute of Fusion Studies, University of Texas, USA

Librarian, Magnetic Fusion Program, Lawrence Livermore National Laboratory, USA

Library, General Atomics, USA

Plasma Physics Group, Fusion Energy Research Program, University of California at San Diego, USA

Plasma Physics Library, Columbia University, USA

Alkesh Punjabi, Center for Fusion Research and Training, Hampton University, USA

Dr. W.M. Stacey, Fusion Research Center, Georgia Institute of Technology, USA

Dr. John Willis, U.S. Department of Energy, Office of Fusion Energy Sciences, USA

Mr. Paul H. Wright, Indianapolis, Indiana, USA 
The Princeton Plasma Physics Laboratory is operated by Princeton University under contract with the U.S. Department of Energy.

\author{
Information Services \\ Princeton Plasma Physics Laboratory \\ P.O. Box 451 \\ Princeton, NJ 08543
}

Phone: 609-243-2750

Fax: 609-243-2751

e-mail: pppl_info@pppl.gov

Internet Address: http://www.pppl.gov 